\title{
Increase of the particle hit rate in a laser single-particle mass spectrome- ter by pulse delayed extraction technology
}

\section{Ying Chen et al.}

Correspondence to: Lei Li (lileishdx@163.com)

The copyright of individual parts of the supplement might differ from the CC BY 4.0 License. 
Below the comparison of SPAMS mass spectra acquired in DC and DE modes are presented. Fig. 1 - Fig. 3 show the spectra obtained using PSL (Polystyrene latex particles), with $266 \mathrm{~nm}$ laser.

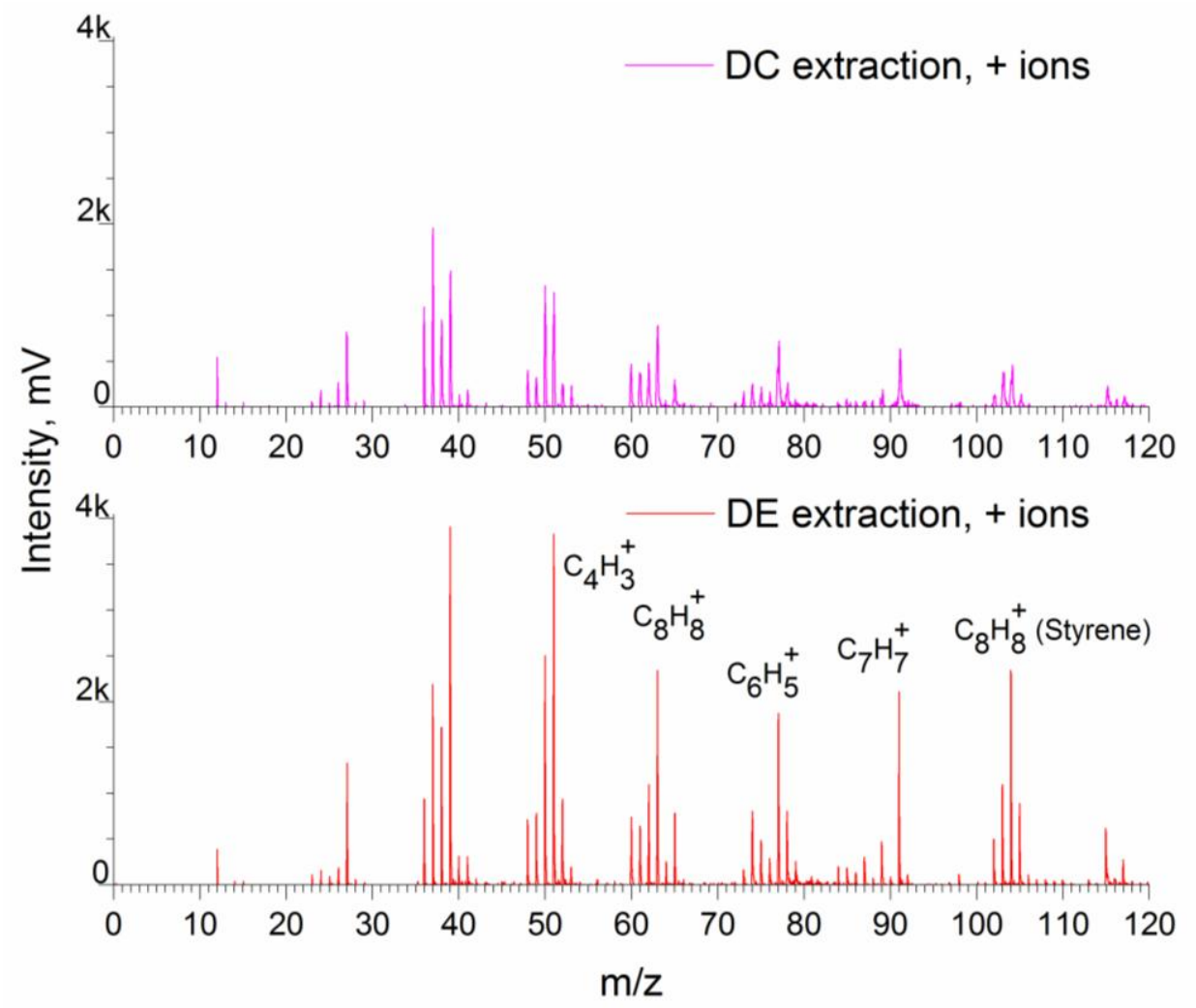

Fig. 1. Comparison of positive ions mass spectra obtained from PSL particles using SPAMS instrument with $D C$ and $D E$ extraction modes, in the mass range $0<m / z<120$.

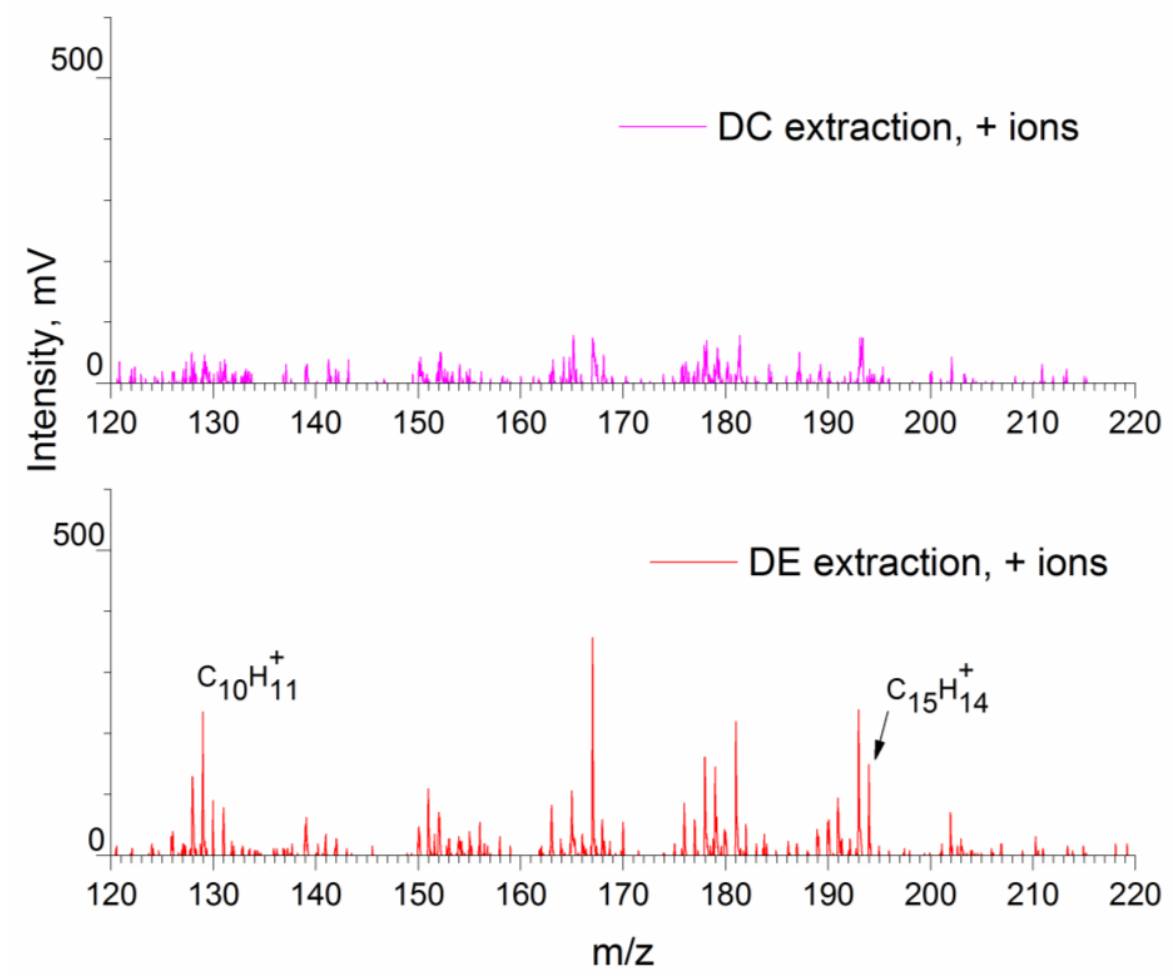

Fig. 2. Comparison of positive ions mass spectra obtained from PSL particles using SPAMS instrument with $D C$ and $D E$ extraction modes, in the mass range $120<m / z<220$. 


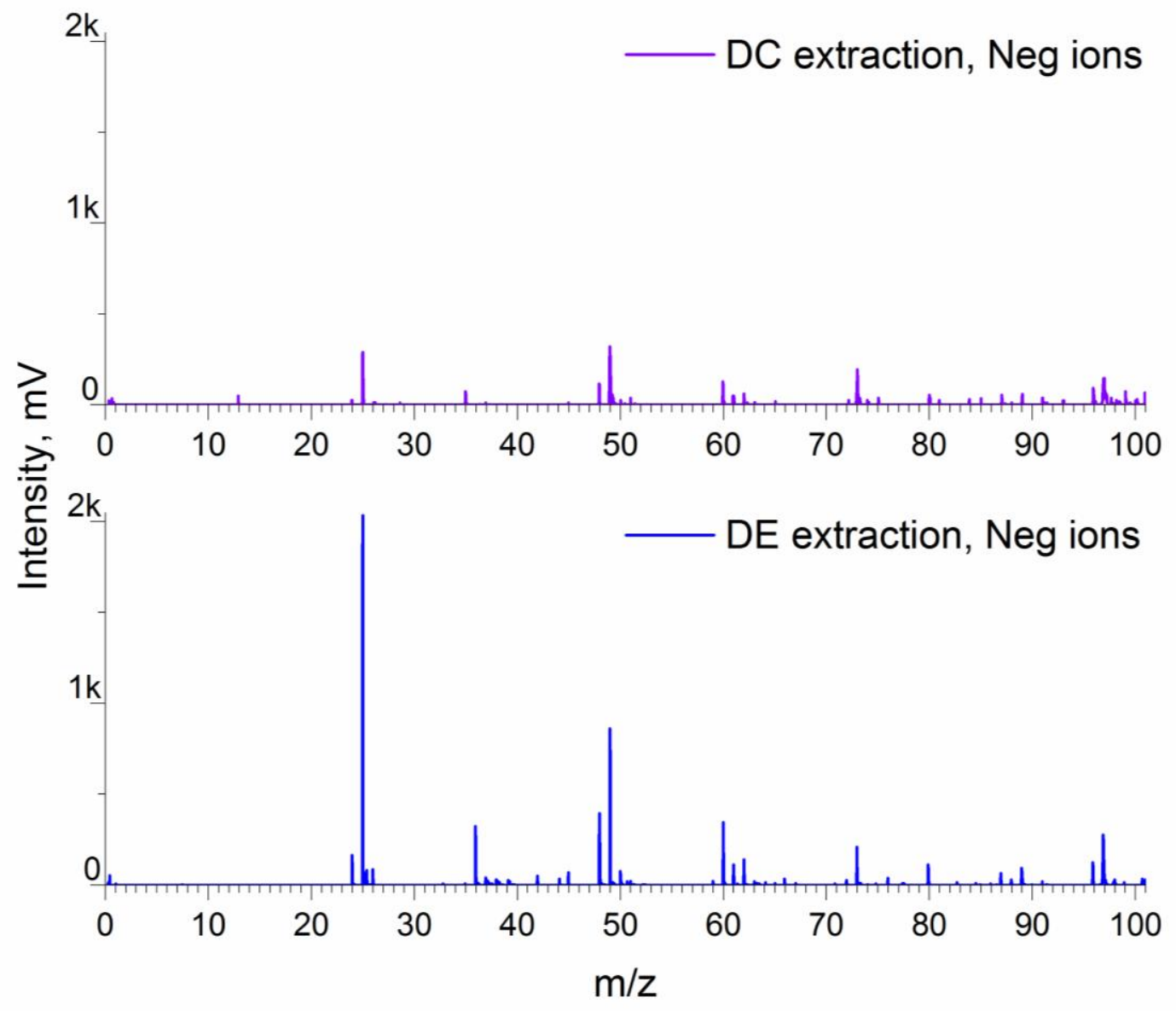

Fig. 3. Comparison of negative ions mass spectra obtained from PSL particles using SPAMS instrument with $D C$ and $D E$ extraction modes, in the mass range $0<m / z<120$.

We can see from these mass spectra, that mass spectra are similar by their peaks sets. There is the significant difference in the peaks intensity. We suppose that this difference is caused mainly by higher mass resolution in case of DE. The relative intensity of some peaks differ in DE and DC cases, presumably by collision energy increase in case of DC extraction. For instance (Fig. 2), we observe $\boldsymbol{C}_{10} \boldsymbol{H}_{11}+$ and $\boldsymbol{C}_{15} \boldsymbol{H}_{14}{ }^{+}$ ions in DE mode, but these dimer fragments of Polystyrene are at the noise level in DC mass spectra. The differences in negative ions mass spectra (Fig. 3) can be explained by the different mechanism of the negative ion formation and a particular features of the electron capture cross-section dependencies.

Also, Fig. 4 shows the comparison of mass spectra of ambient particles. Left side of the figure are the spectra acquired without delay, while the right side are the spectra acquired with delayed extraction, the delay value was $130 \mathrm{~ns}$. Note please, that all ambient particles are different, so, the peaks set for the same kind of particles are similar, but their relative abundances can differ. Negative ions in Fig. 4 are $\mathrm{O}^{-}$, $\mathrm{OH}^{-}, \mathrm{CN}^{-}, \mathrm{NO}_{2}{ }^{-}, \mathrm{NO}_{3}{ }^{-}, \mathrm{HSO}_{4}{ }^{-}$, and positive ions are $\mathrm{C}_{\mathrm{n}} \mathrm{H}_{\mathrm{k}}{ }^{+}$. 


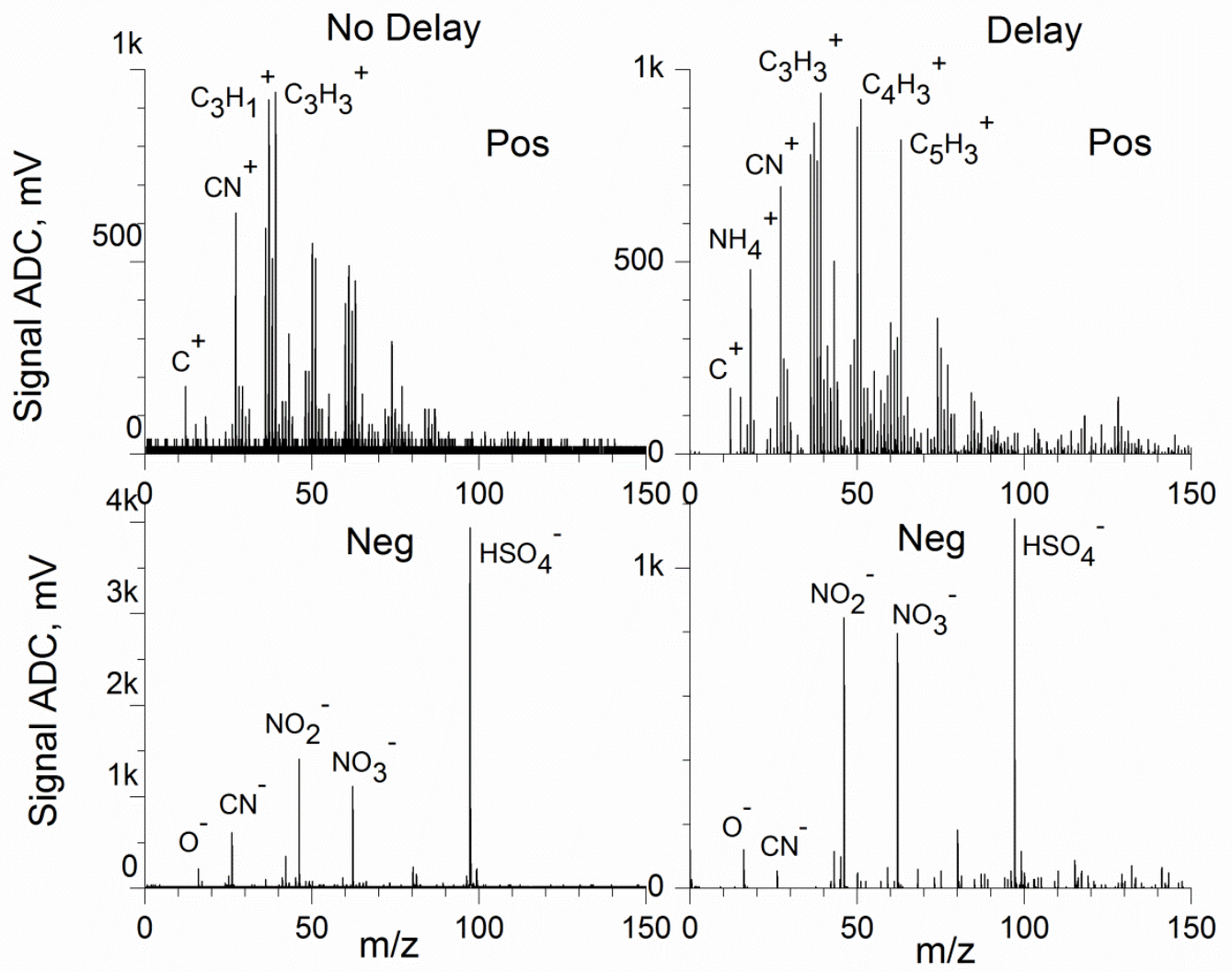

Fig. 4. The comparison of organic-containing single particles mass spectra. Constant field (DC) extraction is on the left, and DE extraction is on the right. 